\title{
Sleep Quality is associated with Central Arterial Stiffness in Postmenopausal Women: A Cross-sectional Pilot Study
}

\author{
Youngju Choi ${ }^{1,2,(\mathbb{D})}$, Nobuhiko Akazawa ${ }^{1,3}$, Asako Zempo-Miyaki ${ }^{1,4}$, Song-Gyu Ra ${ }^{1,5}$, Seiji Maeda ${ }^{1, *}$ (D) \\ ${ }^{1}$ Faculty of Health and Sport Science, University of Tsukuba, Tsukuba, Japan \\ ${ }^{2}$ Institute of Sports \& Arts Convergence, Inha University, Korea \\ ${ }^{3}$ Department of Sports Research, Japan Institute of Sports Sciences, Tokyo, Japan \\ ${ }^{4}$ Faculty of Sports and Health Sciences, Ryutsu Keizai University, Ryugasaki, Japan \\ ${ }^{5}$ Institute of Liberal Arts and Sciences, Tokushima University, Tokushima, Japan
}

\section{ARTICLE INFO}

Article History

Received 28 May 2020

Accepted 28 September 2020

Keywords

Actigraphy

sleep quality

pulse wave velocity

PSQI

\begin{abstract}
This study aimed to investigate the associations between sleep quality and arterial stiffness in healthy postmenopausal women. A total of 31 healthy postmenopausal women aged between 50 and 74 years participated in this study. Objectively and subjectively measured sleep quantity and quality were concomitantly obtained by a waist-worn actigraphy, Pittsburgh Sleep Quality Index (PSQI) questionnaire, and daily sleep diary. Carotid-femoral pulse wave velocity (cfPWV), brachial-ankle PWV (baPWV), and femoral-ankle PWV (faPWV) were measured as indices of arterial stiffness. Based on the PSQI score, the participants were divided into good (PSQI $<5.5 ; n=21$ ) and poor (PSQI $>5.5 ; n=10$ ) sleepers. Self-reported sleep duration was significantly longer in poor sleepers than in good sleepers. However, there was no difference in total sleep time measured by actigraphy between the two groups. Additionally, sleep latency and wake after sleep onset significantly increased, and sleep efficiency significantly decreased in poor sleepers than in good sleepers. The cfPWV and baPWV were significantly higher in poor sleepers than in good sleepers, even after adjustment for risk factors (i.e., age, blood pressure, and physical activity), but no difference in faPWV. These results suggest that decreased sleep quality is associated with an increase in central arterial stiffness in postmenopausal women.
\end{abstract}

(c) 2020 Association for Research into Arterial Structure and Physiology. Publishing services by Atlantis Press International B.V. This is an open access article distributed under the CC BY-NC 4.0 license (http://creativecommons.org/licenses/by-nc/4.0/)

\section{INTRODUCTION}

Accumulating evidence has consistently shown a U-shaped association between self-reported habitual sleep duration and adverse health outcomes, including cardiovascular disease (CVD) risk factors, cardiovascular morbidity, and mortality [1,2]. Data from nationwide surveys worldwide have reported that more than $30 \%$ of adults have sleep problems [3,4]. In particular, previous studies suggest that females had a $41 \%$ higher risk for sleep disturbances than males [5], and it becomes more prevalent in postmenopausal than premenopausal women [6,7]. Moreover, Chair et al. [8] found a significant relationship between subjective sleep quality and CVD risk in postmenopausal women, indicating that poor sleep quality as well as inadequate sleep duration might increase the CVD risk in postmenopausal women.

Arterial stiffness, especially central arterial stiffness, progressively increases with advancing age [9] and is an independent marker for future CVD events and all-cause mortality [10-12]. In women, decreased circulating estrogens with menopause interact with aging process to accelerate an increase in arterial stiffness [13]. While a prospective cohort study has reported that self-reported sleep duration

"Corresponding author.Email: maeda.seiji.gn@u.tsukuba.ac.jp

Peer review under responsibility of the Association for Research into Arterial Structure and Physiology

Data availability statement: The authors confirm that the data supporting the findings

of this study are available within the article. is associated with arterial stiffness in a U-shaped fashion [14], other studies have found an association between only long sleep duration and arterial stiffness [15-17]. Interestingly, several studies have indicated an association of self-reported sleep quality with increased arterial stiffness measured by brachial-ankle pulse wave velocity (baPWV) $[15,18,19]$. These results suggest a possibility of the adverse effect attributed to an increase in arterial stiffness may be due to not only sleep duration but also sleep quality. However, little research has been done to investigate the effects of sleep quality using subjective and objective measures of sleep on arterial stiffness.

This study aimed to investigate the association between sleep quality and arterial stiffness in healthy postmenopausal women who are known to have sleep disturbance and increased arterial stiffness. We hypothesized that postmenopausal women with poor sleep quality had increased central arterial stiffness. To test our hypothesis, we assessed objectively and subjectively measured sleep quality and quantity, and arterial stiffness in a cross-sectional study of healthy postmenopausal women.

\section{MATERIALS AND METHODS}

\subsection{Participants}

A total of 31 healthy and postmenopausal women (aged 50-74 years old) participated in the study. Participants were nonsmokers, 
nonobese, and free of CVD as assessed by medical history. None of the participants were taking cardiovascular-acting medications or hormone replacement therapy. All participants gave written, informed consent to participate. The Ethical Committee of the University of Tsukuba approved this study.

\subsection{Measurements}

\subsubsection{Subjective and objective sleep quantity and quality}

The assessment of sleep quality was determined by using both subjective and objective measures. The Pittsburgh Sleep Quality Index (PSQI) questionnaire is a validated self-administered instrument that measures subjective sleep quality over the previous month. It consists of 18 items that, in turn, are comprised of seven components covering subjective sleep quality, sleep duration, sleep onset, habitual sleep efficiency, sleep disturbances, use of sleeping medications, and daytime dysfunction. Seven component scores are summed to yield a total PSQI score (range, 0-21 points), with higher scores on the PSQI indicating poorer sleep quality. Based on the total PSQI score, participants were divided into two groups: "Good sleepers" with PSQI score of less than 5.5 and "Poor sleepers" with PSQI score of more than 5.5 [20].

Sleep quality was also objectively assessed using actigraphy for seven nights at home (five weekdays and two weekends) (Kenz Lifecoder EX; Suzuken Co. Ltd., Nagoya, Japan). A concomitant daily sleep diary was given to record their bed/rise time and other night-time events. The information derived from the sleep daily was also used to check possible discrepancies or missing data from the actigraphy. The actigraphy data were downloaded to a sleep software program (Kissei Comtec Co. Ltd., Nagano, Japan). They were analyzed to yield variables estimating sleep quality (sleep efficiency and sleep latency) and quantity (total sleep time). The software provides the Enomoto algorithm to estimate sleep parameters from activity counts [21]. The mean agreement rate with the corresponding polysomnography (PSG)-based sleep-wake data was $86.9 \%$. The measures were sleep latency, wake after sleep onset (WASO), total sleep time [sum of the amount of sleep time (min), excluding all-time awake], and sleep efficiency (total sleep time divided by the time in bed, multiplied by 100).

\subsubsection{Pulse wave velocity, blood pressure, and heart rate}

After a minimum resting period of $15 \mathrm{~min}, \mathrm{PWV}$, blood pressure, and heart rate were measured in a quiet, temperature-controlled room $\left(24-26^{\circ} \mathrm{C}\right)$. Carotid-femoral PWV (cfPWV), femoral-ankle PWV (faPWV), and brachial-ankle PWV (baPWV) were measured using a previously described automatic waveform analyzer that previously described (form PWV/ABI; Colin Medical Technology, Komaki, Japan) [22,23]. Electrocardiogram, bilateral brachial, and ankle blood pressures, and carotid and femoral arterial pulse waves were simultaneously measured. The carotid and femoral arterial pulse waves were obtained in triplicate using arterial applanation tonometry, incorporating an array of 15 transducers. Bilateral brachial and ankle blood pressure waveforms were recorded for $10 \mathrm{~s}$ using extremity cuffs connected to an oscillometric pressure sensor wrapped on both arm and ankles. The distance between the two applanation sites was assessed in triplicate with a random zerolength measurement of the surface of the body, using a non-elastic tape. Pulse wave transit time was determined based on the delay in time between the proximal and distal 'foot' waveforms. The foot of the pulse wave was identified as the commencement of the sharp systolic upstroke, which was automatically detected. PWV was calculated as the distance divided by the transit time and was expressed in meters per second.

At the same time as PWV measurement, the resting brachial arterial blood pressure, and heart rate were measured in triplicate using oscillometry and electrocardiogram (form PWV/ABI; Colin Medical Technology).

\subsubsection{Physical activity}

The daily step count was collected in all participants using an accelerometer (Lifecorder; Suzuken Co. Ltd.). Participants were instructed to wear a Lifecorder on their waists continuously for 14 days, except while they were bathing. The data recorded from a continuous 7-day period were used to assess the daily step count of the participants.

\subsubsection{Blood biochemistry}

Blood samples were collected from the antecubital vein in each participant after 12 -h overnight fasting. The serum concentrations of total cholesterol, high-density lipoprotein (HDL) cholesterol, low-density lipoprotein (LDL) cholesterol, and triglyceride and plasma concentration of glucose were determined using standard enzymatic techniques.

\subsection{Statistical Analysis}

The Shapiro-Wilk test was used to assess the normality of all parameters. All values were expressed as mean \pm standard error (SE). The mean differences between good and poor sleepers were examined using independent $t$-test for normally distributes data, or the Mann-Whitney $U$-test for non-normally distributed data (i.e., sleep efficiency). Analysis of Covariance (ANCOVA) was performed to correct the influence of confounding factors of arterial stiffness, such as age, blood pressure, and physical activity. All statistical analyses were performed using IBM SPSS Statistics 22 (SPSS Inc., Chicago, IL, USA), with statistical significance set at $p<0.05$.

\section{RESULTS}

The characteristics of participants divided into good and poor sleepers are shown in Table 1. PSQI and diastolic blood pressure were significantly higher in poor sleepers than in good sleepers (PSQI, good sleepers; $3.4 \pm 0.3$ score vs. poor sleepers; $8.1 \pm 0.4$ score; $p<0.001$; diastolic blood pressure, $70 \pm 2$ vs. $80 \pm 4 \mathrm{mmHg}$; $p<0.05)$. Physical activity measured as daily step counts was significantly higher in good sleepers than in poor sleepers ( $8655 \pm 637$ vs. $5525 \pm 747$ steps; $p<0.005)$. There were no significant differences in 
Table 1 Characteristics of the participants

\begin{tabular}{lccc}
\hline & $\begin{array}{c}\text { Good sleepers } \\
(\boldsymbol{n}=\mathbf{2 1})\end{array}$ & $\begin{array}{c}\text { Poor sleepers } \\
(\boldsymbol{n}=\mathbf{1 0})\end{array}$ & $\boldsymbol{p}$-value \\
\hline PSQI, score & $3.4 \pm 0.3$ & $8.1 \pm 0.4^{* * *}$ & 0.000 \\
Age, years & $60 \pm 1$ & $61 \pm 2$ & 0.744 \\
Height, cm & $154 \pm 1$ & $153 \pm 2$ & 0.415 \\
Weight, kg & $55 \pm 2$ & $53 \pm 3$ & 0.560 \\
Body mass index, kg/m ${ }^{2}$ & $23.0 \pm 0.7$ & $22.7 \pm 1.4$ & 0.827 \\
Total cholesterol, mg/dl & $231 \pm 5$ & $230 \pm 9$ & 0.93 \\
HDL cholesterol, mg/dl & $67 \pm 4$ & $69 \pm 8$ & 0.833 \\
LDL cholesterol, mg/dl & $138 \pm 6$ & $142 \pm 9$ & 0.737 \\
Triglycerides, mg/dl & $116 \pm 18$ & $95 \pm 13$ & 0.472 \\
Glucose, mg/dl & $92 \pm 1$ & $91 \pm 2$ & 0.769 \\
Systolic blood pressure, mmHg & $117 \pm 3$ & $126 \pm 5$ & 0.141 \\
Diastolic blood pressure, mmHg & $70 \pm 2$ & $80 \pm 4$ & 0.012 \\
Heart rate, beats/min & $60 \pm 2$ & $64 \pm 3$ & 0.161 \\
Daily Step counts, steps & $8655 \pm 637$ & $5525 \pm 747^{* *}$ & 0.004 \\
\hline
\end{tabular}

" $p<0.05$ vs. good sleepers; " $p<0.005$ vs. good sleepers; " $p<0.001$ vs. good sleepers;

Values are mean $+\mathrm{SE}$.

Table 2 Sleep parameters of good sleepers and poor sleepers

\begin{tabular}{lcc}
\hline Variable & $\begin{array}{c}\text { Good sleepers } \\
(\boldsymbol{n}=\mathbf{2 1})\end{array}$ & $\begin{array}{c}\text { Poor sleepers } \\
(\boldsymbol{n}=\mathbf{1 0})\end{array}$ \\
\hline $\begin{array}{l}\text { Self-reported } \\
\quad \text { Sleep duration, min }\end{array}$ & $387 \pm 11$ & $436 \pm 16^{*}$ \\
Actigraphy & & \\
$\quad$ Sleep latency, min & $6 \pm 1$ & $10 \pm 1^{*}$ \\
$\quad$ Wake after sleep onset, min & $59 \pm 8$ & $91 \pm 12^{*}$ \\
$\quad$ Total sleep time, min & $319 \pm 8$ & $328 \pm 15$ \\
$\quad$ Sleep efficiency, \% & $80.8 \pm 1.9$ & $75.0 \pm 2.6^{*}$ \\
\hline
\end{tabular}

" $p<0.05$ vs. good sleepers. Values are means \pm SE.

age, BMI, systolic blood pressure, heart rate, total cholesterol, HDL cholesterol, LDL cholesterol, triglyceride, and glucose between the two groups.

Sleep parameters, as assessed by actigraphy and sleep daily, are shown in Table 2. Significant differences were found in self-reported sleep duration, sleep latency, WASO, and sleep efficiency between two groups. Poor sleepers spent 49 more minutes asleep per night (387 \pm 11 vs. $436 \pm 16 \mathrm{~min} ; p<0.05$ ) based on the self-reported sleep duration. However, based on actigraphy, total sleep time did not differ in both groups ( $319 \pm 8$ vs. $328 \pm 15 \mathrm{~min} ; p=0.27$ ). Furthermore, in poor sleepers, sleep latency was 4 min longer $(6 \pm 1$ vs. $10 \pm 1 \mathrm{~min}$; $p<0.05)$, WASO was $32 \mathrm{~min}$ greater (59 \pm 8 vs. $91 \pm 12 \mathrm{~min} ; p<$ $0.05)$, and sleep efficiency was $5.8 \%$ worse $(80.8 \pm 1.9$ vs. $75.0 \pm 2.6 \%$; $p<0.05)$ compared to good sleepers.

Arterial stiffness measured by PWV between the two groups is shown in Figure 1. The cfPWV, faPWV, and baPWV in poor sleepers were found to be significantly higher than that in good sleepers. Furthermore, in ANCOVA analyses adjusted for age, blood pressure, and physical activity, the results of cfPWV and baPWV remained significant (adjusted cfPWV, $9.3 \pm 0.2$ vs. $10.5 \pm 0.4 \mathrm{~m} / \mathrm{s} ; F=6.34$, $p<0.05$; adjusted baPWV, $13.0 \pm 0.3$ vs. $14.3 \pm 0.4 \mathrm{~m} / \mathrm{s} ; F=5.14$, $p<0.05$ ) (Figure 1). However, there was no significant difference in faPWV between the two groups (adjusted faPWV, $8.2 \pm 0.2$ vs. $8.6 \pm 0.3 \mathrm{~m} / \mathrm{s} ; p=0.26$ ).
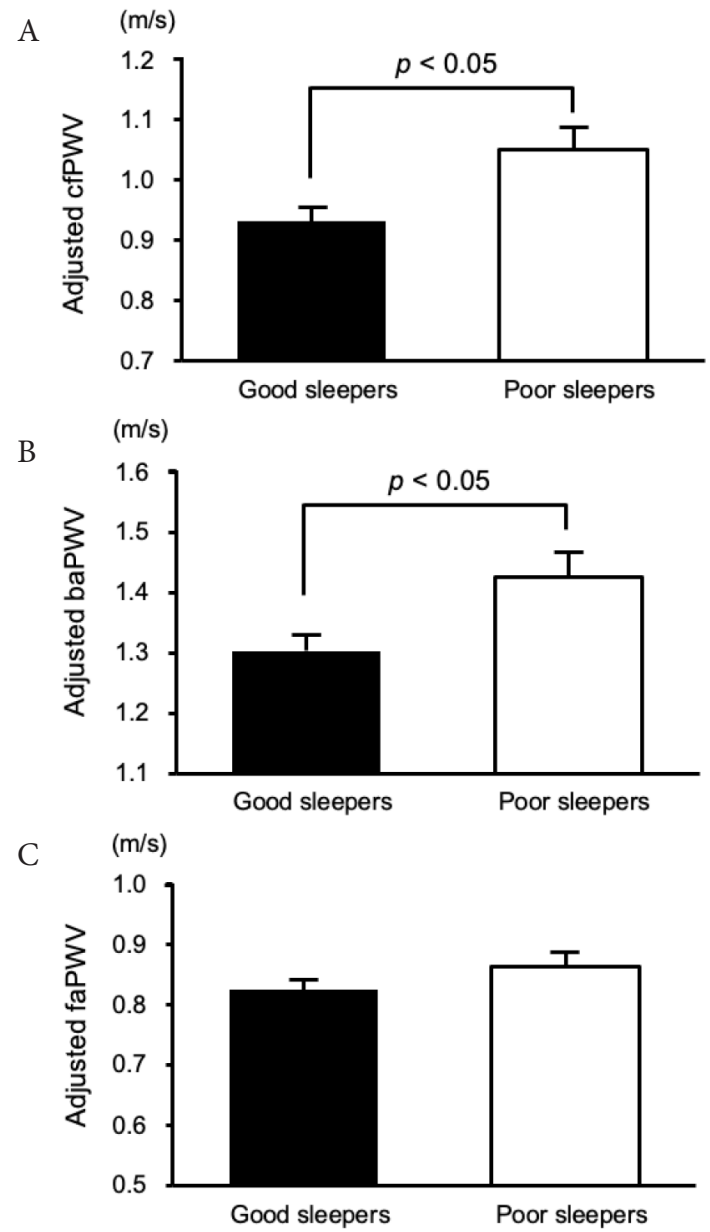

Figure 1 Carotid-femoral PWV (A), brachial-ankle PWV (B), and femoral-ankle PWV (C) values for good sleepers and poor sleepers. Differences in PWV between good sleepers and poor sleepers were assessed by a covariance analysis model that included age, blood pressure, and physical activity as covariates. Data are expressed as mean \pm SE.

\section{DISCUSSION}

The present study examined the effects of sleep quality on arterial stiffness in healthy postmenopausal women. The main finding of this study was that postmenopausal women with poor sleep quality had significantly higher cfPWV and baPWV, but not faPWV, even after adjustment for age, blood pressure, and physical activity. These results suggest that sleep quality could play an essential role in central arterial stiffness in postmenopausal women. This study added new scientific evidence concerning the relationship between sleep quality and arterial stiffness by using an objective and standardized method of measuring sleep activity (i.e., actigraphy) and included the analysis of potential confounders.

With respect to the association between sleep and arterial stiffness, subjective tools, such as self-reported questionnaires, have been used as a simple method for assessing sleep behavior [14-19]. However, those of previous studies have reported inconsistent associations of self-reported sleep with arterial stiffness. This methodology only assesses subjective sleep quantity or quality without capturing other components, and subjective sleep questionnaires may not 
correspond well with objective sleep estimates. Indeed, Lauderdale et al. [24] revealed that self-reported sleep duration has a moderate, not high, correlation with actigraphy-measured sleep, indicating the importance of adding objective recordings (e.g., actigraphy) under natural environmental conditions. Actigraphy provides objective sleep parameters, such as sleep latency, WASO, total sleep time, and sleep efficiency. There have been a few studies that have reported association of objective sleep variable with arterial stiffness $[25,26]$, the available evidence may be not applicable to postmenopausal women with high risk of poor sleep quality. To the best of our knowledge, this is the first report regarding the association between sleep quality and arterial stiffness using subjective and objective measured sleep especially in postmenopausal women.

This study showed that individuals who have poor sleep quality with a PSQI score $>5.5$, did have a longer self-reported sleep duration than their counterparts who have good sleep quality (7.3 vs. $6.5 \mathrm{~h}$ ). In turn, the poor sleepers spent more time in bed than the good sleepers. However, there was no difference in total sleep time, resulting in a reduction in sleep efficiency as estimated by actigraphy in the poor sleepers. Although an epidemiologic study showed an association between self-reported habitual sleep duration and arterial stiffness [14], several studies using subjective measures of sleep duration have failed to find an association of short or long sleep duration with arterial stiffness in women [27-29]. In this study, the self-reported daily sleep durations of good and poor sleepers were 6.5 and $7.3 \mathrm{~h}$, which approximates the empirical evidence, showing that adults require, on average, approximately seven hours of sleep for lower arterial stiffness. Interestingly, this study revealed that individuals who had poor sleep quality while having appropriate self-reported sleep duration had significantly increased arterial stiffness even after adjustment for potential confounders. Taken together, this study suggests that good sleep quality, as well as adequate sleep duration, may contribute to decreasing arterial stiffness.

Another issue apparent from our findings is that poor sleepers did have a decrease in physical activity. Interestingly, recent studies revealed a bidirectional association between sleep and physical activity, and that sleep quality increases physical activity, and higher levels of physical activity promote good sleep [30,31]. Furthermore, evidence from our group and other studies has shown that physical activity and aerobic exercise lead to decreased arterial stiffness in postmenopausal women [32-36]. Because this study was a cross-sectional design, we could not consider the possible influence of physical activity related to decreasing arterial stiffness. Nevertheless, our results clearly show that individuals who had poor sleep quality had significantly increased arterial stiffness even after adjustment for physical activity, suggesting that adequate sleep in postmenopausal women has a beneficial effect on reducing arterial stiffness. Further studies are needed as direct evidence (e.g., the role of sleep parameters, such as sleep stages on arterial stiffness) to support these results.

Unfortunately, our results do not fully elucidate the mechanisms underlying the association of sleep quality with arterial stiffness in postmenopausal women. Given that endothelial function affects the regulation of arterial stiffness [37,38], impaired endothelial function induced by poor sleep quality may predispose to increased arterial stiffness. Several studies have reported the association of reduced endothelial function with decreased self-reported sleep quality (decreased PSQI scores) [39], \% Rapid Eye Movement (REM) sleep [40], and increased daytime sleepiness (i.e., daytime dysfunction) [39]. Increased inflammation, which may lead to arterial stiffening and endothelial dysfunction [41-43], was also associated with poor sleep quality and circadian disruption [44,45]. Friedman et al. [45] reported that better sleep efficiency was related to lower levels of IL-6 in postmenopausal women. In addition, a laboratory-based study by Wright et al. [44] examined whether chronic circadian misalignment, which is related to sleep problems such as poor sleep and daytime sleepiness could affect plasma concentrations of pro-inflammatory proteins. They showed that weeks of circadian misalignment increased levels of TNF- $\alpha$ and CRP, with subsequent disrupted sleep in healthy adults. Interestingly, a recent study has shown that circadian rhythm-related gene (i.e., PER2) decreased as a consequence of menopause [46]. Furthermore, their study reported that menopause-induced oxidative stress, which also has detrimental effects on arterial stiffening [47], parallels a disruption of circadian rhythm clock. Thus, it is possible that reduced endothelial function, increased inflammation, oxidative stress, and circadian disruption caused by low sleep quality might lead to arterial stiffening in postmenopausal women. Additional studies are needed to clarify the mechanisms underlying the impacts of sleep quality on arterial stiffness in postmenopausal women.

Our study had certain limitations. First, cross-sectional design of the study did not allow inference of the causal relationship between sleep quality and arterial stiffness. Second, this study was conducted with a small sample size. Finally, this study included only healthy postmenopausal women. Therefore, the findings of this study cannot be generalized to other populations (i.e., obese individuals, young adults).

\section{CONCLUSION}

In summary, our findings reveal that postmenopausal women with poor sleep quality have higher central arterial stiffness than women with good sleep quality. These findings indicate that the associations of sleep quality with arterial stiffness are important issues in postmenopausal women.

\section{CONFLICTS OF INTEREST}

The authors declare they have no conflicts of interest.

\section{AUTHORS' CONTRIBUTION}

YC and SM contributed to conceptualization and funding acquisition. NA, ZA and SR contributed to data curation and formal analysis. YC and SM contributed in writing (original draft and review \& editing) the manuscript.

\section{FUNDING}

This work was supported by Grants-in-Aid for Japan Society for the Promotion of Science (JSPS) Research Fellows Grant Number 2503006 . 


\section{REFERENCES}

[1] Cappuccio FP, D’Elia L, Strazzullo P, Miller MA. Quantity and quality of sleep and incidence of type 2 diabetes: a systematic review and meta-analysis. Diabetes Care 2010;33:414-20.

[2] Cappuccio FP, Cooper D, D’Elia L, Strazzullo P, Miller MA. Sleep duration predicts cardiovascular outcomes: a systematic review and meta-analysis of prospective studies. Eur Heart J 2011;32:1484-92.

[3] Wilsmore BR, Grunstein RR, Fransen M, Woodward M, Norton R, Ameratunga S. Sleep habits, insomnia, and daytime sleepiness in a large and healthy community-based sample of New Zealanders. J Clin Sleep Med 2013;9:559-66.

[4] Furihata R, Uchiyama M, Takahashi S, Suzuki M, Konno C, Osaki $\mathrm{K}$, et al. The association between sleep problems and perceived health status: a Japanese nationwide general population survey. Sleep Med 2012;13:831-7.

[5] Phillips BA, Collop NA, Drake C, Consens F, Vgontzas AN, Weaver TE. Sleep disorders and medical conditions in women. Proceedings of the Women \& Sleep Workshop, National Sleep Foundation, Washington, DC, March 5-6, 2007. J Womens Health (Larchmt) 2008;17:1191-9.

[6] Sun D, Shao H, Li C, Tao M. Sleep disturbance and correlates in menopausal women in Shanghai. J Psychosom Res 2014;76:237-41.

[7] Young T, Rabago D, Zgierska A, Austin D, Finn L. Objective and subjective sleep quality in premenopausal, perimenopausal, and postmenopausal women in the Wisconsin Sleep Cohort Study. Sleep 2003;26:667-72.

[8] Chair SY, Wang Q, Cheng HY, Lo SWS, Li XM, Wong EML, et al. Relationship between sleep quality and cardiovascular disease risk in Chinese post-menopausal women. BMC Womens Health 2017;17:79.

[9] Mitchell GF, Parise H, Benjamin EJ, Larson MG, Keyes MJ, Vita JA, et al. Changes in arterial stiffness and wave reflection with advancing age in healthy men and women: the Framingham Heart Study. Hypertension 2004;43:1239-45.

[10] Willum-Hansen T, Staessen JA, Torp-Pedersen C, Rasmussen S, Thijs L, Ibsen H, et al. Prognostic value of aortic pulse wave velocity as index of arterial stiffness in the general population. Circulation 2006;113:664-70.

[11] Mitchell GF, Hwang SJ, Vasan RS, Larson MG, Pencina MJ, Hamburg NM, et al. Arterial stiffness and cardiovascular events the Framingham Heart Study. Circulation 2010;121;505-11.

[12] Meaume S, Benetos A, Henry OF, Rudnichi A, Safar ME. Aortic pulse wave velocity predicts cardiovascular mortality in subjects $>70$ years of age. Arterioscler Thromb Vasc Biol 2001;21:2046-50.

[13] Takahashi K, Miura S, Mori-Abe A, Kawagoe J, Takata K, Ohmichi M, et al. Impact of menopause on the augmentation of arterial stiffness with aging. Gynecol Obstet Inves 2005;60:162-6.

[14] Wolff B, Volzke H, Schwahn C, Robinson D, Kessler C, John U. Relation of self-reported sleep duration with carotid intimamedia thickness in a general population sample. Atherosclerosis 2008;196:727-32.

[15] Kim CW, Chang Y, Zhao D, Cainzos-Achirica M, Ryu S, Jung HS, et al. Sleep duration, sleep quality, and markers of subclinical arterial disease in healthy men and women. Arterioscler Thromb Vasc Biol 2015;35:2238-45.
[16] Abe T, Aoki T, Yata S, Okada M. Sleep duration is significantly associated with carotid artery atherosclerosis incidence in a Japanese population. Atherosclerosis 2011;217:509-13.

[17] Niijima S, Nagai M, Hoshide S, Takahashi M, Shimpo M, Kario $\mathrm{K}$, et al. Long sleep duration: a nonconventional indicator of arterial stiffness in Japanese at high risk of cardiovascular disease: the J-HOP study. J Am Soc Hypertens 2016;10:429-37.

[18] Zhou Y, Yang R, Li C, Tao M. Sleep disorder, an independent risk associated with arterial stiffness in menopause. Sci Rep 2017;7:1904.

[19] Osonoi Y, Mita T, Osonoi T, Saito M, Tamasawa A, Nakayama $S$, et al. Poor sleep quality is associated with increased arterial stiffness in Japanese patients with type 2 diabetes mellitus. Bmc Endocr Disord 2015;15:29.

[20] Buysse DJ, Reynolds CF, Monk TH, Berman SR, Kupfer DJ. The Pittsburgh Sleep Quality Index: a new instrument for psychiatric practice and research. Psychiatry Res 1989;28:193-213.

[21] Enomoto M, Endo T, Suenaga K, Miura N, Nakano Y, Kohtoh S, et al. Newly developed waist actigraphy and its sleep/wake scoring algorithm. Sleep Biol Rhythms 2009;7:17-22.

[22] Sugawara J, Hayashi K, Yokoi T, Cortez-Cooper MY, DeVan AE, Anton MA, et al. Brachial-ankle pulse wave velocity: an index of central arterial stiffness? J Hum Hypertens 2005;19:401-6.

[23] Kumagai H, Yoshikawa T, Myoenzono K, Kosaki K, Akazawa N, Asako ZM, et al. Sexual function is an indicator of central arterial stiffness and arterial stiffness gradient in Japanese adult men. J Am Heart Assoc 2018;7:e007964.

[24] Lauderdale DS, Knutson KL, Yan LL, Liu K, Rathouz PJ. Selfreported and measured sleep duration: how similar are they? Epidemiology 2008;19:838-45.

[25] Ma CC, Burchfiel CM, Charles LE, Dorn JM, Andrew ME, Gu JK, et al. Associations of objectively measured and self-reported sleep duration with carotid artery intima media thickness among police officers. Am J Ind Med 2013;56:1341-51.

[26] Kadoya M, Kurajoh M, Kakutani-Hatayama M, Morimoto A, Miyoshi A, Kosaka-Hamamoto K, et al. Low sleep quality is associated with progression of arterial stiffness in patients with cardiovascular risk factors: HSCAA study. Atherosclerosis 2018;270:95-101.

[27] Tsai TC, Wu JS, Yang YC, Huang YH, Lu FH, Chang CJ. Long sleep duration associated with a higher risk of increased arterial stiffness in males. Sleep 2014;37:1315-20.

[28] Anujuo K, Stronks K, Snijder MB, Jean-Louis G, van den Born BJ, Peters RJ, et al. Relationship between sleep duration and arterial stiffness in a multi-ethnic population: the HELIUS study. Chronobiol Int 2016;33:543-52.

[29] Yoshioka E, Saijo Y, Kita T, Okada E, Satoh H, Kawaharada M, et al. Relation between self-reported sleep duration and arterial stiffness: a cross-sectional study of middle-aged Japanese civil servants. Sleep 2011;34:1681-6.

[30] Rayward AT, Burton NW, Brown WJ, Holliday EG, Plotnikoff RC, Duncan MJ. Associations between changes in activity and sleep quality and duration over two years. Med Sci Sport Exer 2018;50:2425-32.

[31] Kline CE. The bidirectional relationship between exercise and sleep: implications for exercise adherence and sleep improvement. Am J Lifestyle Med 2014;8:375-9.

[32] Matsubara T, Miyaki A, Akazawa N, Choi Y, Ra SG, Tanahashi $\mathrm{K}$, et al. Aerobic exercise training increases plasma Klotho levels 
and reduces arterial stiffness in postmenopausal women. Am J Physiol Heart Circ Physiol 2014;306:H348-H55.

[33] Tanahashi K, Akazawa N, Miyaki A, Choi Y, Ra SG, Matsubara $\mathrm{T}$, et al. Aerobic exercise training decreases plasma asymmetric dimethylarginine concentrations with increase in arterial compliance in postmenopausal women. Am J Hypertens 2014;27:415-21.

[34] Yoshizawa M, Maeda S, Miyaki A, Misono M, Choi Y, Shimojo $\mathrm{N}$, et al. Additive beneficial effects of lactotripeptides and aerobic exercise on arterial compliance in postmenopausal women. Am J Physiol Heart Circ Physiol 2009;297:H1899-H903.

[35] Sugawara J, Otsuki T, Tanabe T, Hayashi K, Maeda S, Matsuda M. Physical activity duration, intensity, and arterial stiffening in postmenopausal women. Am J Hypertens 2006;19:1032-6.

[36] Moreau KL, Donato AJ, Seals DR, DeSouza CA, Tanaka H. Regular exercise, hormone replacement therapy and the age-related decline in carotid arterial compliance in healthy women. Cardiovasc Res 2003;57:861-8.

[37] Wilkinson IB, MacCallum H, Cockcroft JR, Webb DJ. Inhibition of basal nitric oxide synthesis increases aortic augmentation index and pulse wave velocity in vivo. Br J Clin Pharmacol 2002;53:189-92.

[38] McEniery CM, Qasem A, Schmitt M, Avolio AP, Cockcroft JR, Wilkinson IB. Endothelin-1 regulates arterial pulse wave velocity in vivo. J Am Coll Cardiol 2003;42:1975-81.

[39] Behl M, Bliwise D, Veledar E, Cunningham L, Vazquez J, Brigham $\mathrm{K}$, et al. Vascular endothelial function and self-reported sleep. Am J Med Sci 2014;347:425-8.
[40] Cooper DC, Ziegler MG, Milic MS, Ancoli-Israel S, Mills PJ, Loredo JS, et al. Endothelial function and sleep: associations of flow-mediated dilation with perceived sleep quality and rapid eye movement (REM) sleep. J Sleep Res 2014;23:84-93.

[41] Trepels T, Zeiher AM, Fichtlscherer S. The endothelium and inflammation. Endothelium 2006;13:423-9.

[42] Mattace-Raso FU, van der Cammen TJ, van der Meer IM, Schalekamp MA, Asmar R, Hofman A, et al. C-reactive protein and arterial stiffness in older adults: the Rotterdam Study. Atherosclerosis 2004;176:111-16.

[43] Yasmin Y, McEniery C, Wallace S, Mackenzie I, Cockcroft J, Wilkinson IB. C-reactive protein is associated with arterial stiffness in apparently healthy individuals. J Hypertens 2004;22:S298.

[44] Wright Jr KP, Drake AL, Frey DJ, Fleshner M, Desouza CA, Gronfier C, et al. Influence of sleep deprivation and circadian misalignment on cortisol, inflammatory markers, and cytokine balance. Brain Behav Immun 2015;47:24-34.

[45] Friedman EM, Hayney MS, Love GD, Urry HL, Rosenkranz MA, Davidson RJ, et al. Social relationships, sleep quality, and interleukin-6 in aging women. Proc Natl Acad Sci U S A 2005;102:18757-62.

[46] Rangel-Zuñiga OA, Cruz-Teno C, Haro C, Quintana-Navarro GM, Camara-Martos F, Perez-Martinez P, et al. Differential menopause- versus aging-induced changes in oxidative stress and circadian rhythm gene markers. Mech Ageing Dev 2017;164:41-8.

[47] Hildreth KL, Kohrt WM, Moreau KL. Oxidative stress contributes to large elastic arterial stiffening across the stages of the menopausal transition. Menopause 2014;21:624-32. 